\title{
O CONHECIMENTO PRODUZIDO NO PROGRAMA DE MESTRADO DE ENFERMAGEM EM SAÚDE PÚBLICA DA ESCOLA DE ENFERMAGEM DE RIBEIRÃO PRETO-USP E SUAS RELAÇÕES COM AS PRÁTICAS DE SAÚDE
}

\author{
Maria Cecília Puntel de Almeida* \\ Eliete Maria Silva** \\ Tereza Cristina Scatena Villa** \\ Marluce Maria Araújo Assis*** \\ Maria Lúcia Rímoli Kemura****
}

ALMEIDA, M.C.P.de; SILVA, E.M.; VILLA, T.C.S.; ASSIS, M.M.A.; KEMURA, M.L.R. O conhecimento produzido no programa de mestrado de enfermagem em saúde pública da Escola de Enfermagem de Ribeirão Preto-USP e suas relações com as práticas de saúde. Rev.latino-am.enfermagem, Ribeirão Preto, v. 8, n. 5, p. 91-96, outubro 2000.

O artigo objetiva analisar os objetos de pesquisa das dissertações do Programa de Mestrado de Enfermagem em Saúde Pública do MISP da EERP-USP e suas relações com os problemas oriundos das práticas de saúde. Estas, foram classificadas em seis áreas temáticas: Organização da Assistência à Saúde, Promoção da Saúde, Processo Saúde-Doença, Nutrição-Alimentação, Prática Profissional e Imunologia. Destaca-se a contribuição de trabalhos que abordam a crise dos paradigmas na saúde, as relações de gênero e os movimentos sociais em saúde. Conclui-se que as temáticas retratam as realidades profissionais vivenciadas pelos alunos com propostas de intervenção nos micro-espaços das práticas de saúde.

UNITERMOS: enfermagem em saúde pública, educação de pós-graduação em enfermagem, prática de saúde pública

\section{INTRODUÇÃO}

O Programa de Mestrado de Enfermagem em Saúde Pública foi criado na Escola de Enfermagem de Ribeirão Preto (EERP) da Universidade de São Paulo (USP), pelo Departamento de Enfermagem MaternoInfantil e Saúde Pública (MISP) no ano de 1991, cinco anos após a constituição do departamento.

Os cursos de pós-graduação stricto sensu tiveram início no país na década de setenta, sendo que o primeiro programa de enfermagem, no nível de mestrado foi criado em 1972, na Universidade Federal do Rio de Janeiro (UFRJ) - Escola de Enfermagem Anna Nery e o doutorado no ano de 1981, através do Programa Interunidades de Doutorado das Escolas de Enfermagem de São Paulo e Ribeirão Preto da USP. Atualmente contamos com quinze programas de mestrado e seis de doutorado (ALMEIDA et al., 1995).
O Departamento de Enfermagem Materno-Infantil e Saúde Pública vem preparando e qualificando seus docentes e profissionais da área da saúde, com intuito de desenvolver e aprofundar os conhecimentos em saúde coletiva, e preparando seus funcionários para dar suporte a estas atividades. Suas produções científicas vêm se constituindo em linhas de pesquisa, estão organizadas em núcleos e grupos que se articulam com os serviços de saúde, contando com a participação de outros docentes da área da saúde, profissionais de serviço e alunos de graduação e pósgraduação.

O Relatório de Avaliação de Pós-Graduação da Área de Enfermagem da fundação Coordenadoria de Aperfeiçoamento de Pessoal de Nível Superior - CAPES, relativo ao biênio 1985/86, enfatizava que mudanças vinham ocorrendo na sociedade brasileira com o processo constituinte que culminaria com a aprovação da Constituição de 1988 e das diretrizes oriundas da Reforma

\footnotetext{
* Professora Titular do Departamento de Enfermagem Materno-Infantil e Saúde Pública da Escola de Enfermagem de Ribeirão Preto da Universidade de São Paulo

** Professor Doutor do Departamento de Enfermagem Materno-Infantil e Saúde Pública da Escola de Enfermagem de Ribeirão Preto da Universidade de São Paulo

*** Doutoranda da Programa Interunidades de Doutoramento da Escola de Enfermagem de Ribeirão Preto/Escola de Enfermagem da Universidade de São Paulo

**** Enfermeira da Secretaria Municipal de Saúde de Ribeirão Preto
} 
Sanitária, em processo no país, bem como a necessidade de desenvolver ampla discussão e reflexão sobre a assistência de enfermagem praticada no Brasil. O relatório explicitava que esta discussão poderia nos levar a novos perfis profissionais, exigindo cuidadosa análise da prática de enfermagem articulada ao desenvolvimento técnicocientífico, enquanto parte do processo de mudanças sóciopolítico-econômicas do país.

Concomitante a este processo de mobilização e responsabilização do setor saúde, no âmbito nacional, os docentes juntamente com os profissionais do município e da região participavam (e continuam participando) do movimento de transformações dos serviços de saúde, bem como, das discussões acerca da necessidade de produzir novos conhecimentos que possibilitassem repensar e intervir nas práticas de enfermagem e de saúde.

Assim, sensível a esta inquietação existente no cenário nacional e contando com uma pesquisa já organizada em núcleos temáticos, o Departamento de Enfermagem Materno-Infantil e Saúde Pública iniciou um processo de reflexão sobre o seu compromisso frente à profissão e à saúde da população brasileira, os recursos disponíveis que pudessem contribuir para o avanço das soluções dos problemas de saúde coletiva, que culminou com a organização do Programa de Mestrado de Enfermagem em Saúde Pública.

O objetivo do Programa é qualificar profissionais de saúde com formação para: o ensino superior de enfermagem, a pesquisa em saúde coletiva, a análise crítica do conhecimento específico da área e de suas práticas, tendo em vista as necessidades do setor saúde no país (ESCOLA DE ENFERMAGEM DE RIBEIRÃO PRETO, 1990).

Segundo MORYIA et al. (1998),

"a enfermagem é uma das práticas profissionalizantes da área da saúde e como tal, os objetos de investigação têm sido predominantemente termos oriundos deste trabalho, como os relativos ao cuidado e assistência de enfermagem; à organização e administração dessa assistência; aos trabalhos educativos e de promoção à saúde; aos recursos humanos em enfermagem; ao ensino; aos estudos da própria profissão, como seu papel, seu status, transformações histórico-sociais e trabalho voltados para a promoção humana incluindo o exercício da cidadania" (p.64).

Por assinalarmos sua natureza aplicada, não significa que compactuamos com uma visão utilitarista da produção científica; quer dizer, apesar de estar sempre presente a relação teoria prática, não se avalia a importância da pesquisa estritamente em função de sua aplicação imediata. Por outro lado, a produção de conhecimento possibilita um repensar constante da prática e dos saberes na área de saúde.

A reunião Panamericana de Estudos de PósGraduação em Enfermagem ocorrida em Bogotá, em 1995 (JEFFRIES, 1996), analisa os estudos de pós-graduados e a pesquisa nas Américas apontando que

"enfermeiras melhor preparadas poderão compreender o complexo contexto da saúde, seus problemas ambientais, políticos, éticos, econômicos, humanos, participando na busca de soluções adequadas às realidades específicas de cada país e comunidade. A PósGraduação cria espaços e relações horizontais para fortalecer o trabalho em equipes interdisciplinares que investiguem e atuem em diferentes contextos e situações" (WRIGTH \& ALARCÓN, 1996, p.44).

Justifica-se o trabalho pois o acompanhamento do produto científico da pós-graduação permite avaliar parte do conhecimento e redirecioná-lo segundo os objetivos dos programas e as necessidades de saúde que vão se conformando no cenário nacional e nos regionais. Assim este trabalho tem por objetivo analisar os objetos de pesquisa das dissertações de mestrado de enfermagem em saúde pública do Departamento de Enfermagem Materno-Infantil e Saúde Pública quanto às suas relações com os problemas oriundos das práticas de saúde.

\section{METODOLOGIA}

Para a realização do estudo utilizamos como fonte de coleta de dados o total de dissertações de mestrado defendidas, desde a criação do curso em 1991, até dezembro de 1997, totalizando 31 trabalhos.

Todas as dissertações foram lidas observando-se as características centrais do objeto de investigação, o campo da pesquisa, os objetivos e o quadro metodológico. Em relação aos mestrandos foram levantados os dados sobre: a categoria profissional, a região geográfica de origem, o vínculo institucional e a atividade profissional. Esta caracterização correspondeu não só àqueles que defenderam a dissertação mas também aos que ainda estão cursando. Isto possibilitou fazer uma análise mais abrangente da totalidade dos alunos. Os dados foram complementados através de informações adquiridas no Departamento de Enfermagem Materno-Infantil e Saúde Pública e na Seção de Pós-graduação da EERP-USP.

A ordenação dos dados possibilitou classificar as dissertações em seis áreas temáticas: organização da assistência em saúde, processo saúde-doença, promoção da saúde, nutrição/alimentação, prática 
profissional e imunologia. Esta classificação foi realizada detendo-se nos conteúdos, ou seja, nas distintas apreensões do objeto, dos objetivos do estudo e da metodologia, como também, a interação destas áreas com as áreas de conhecimento do departamento (Enfermagem em Saúde da Mulher, Enfermagem em Saúde da Criança e do Adolescente e Enfermagem em Saúde Pública).

\section{RESULTADOS E DISCUSSÃO}

\subsection{Caracterização dos alunos do programa de mestrado de enfermagem em saúde pública}

Na Tabela 1 pode ser observado que foram matriculados 67 alunos no Curso de Mestrado em Saúde Pública, segundo a categoria profissional, no período de 1991 a 1997. Verifica-se o predomínio do profissional enfermeiro $(74,60 \%)$ durante todo o período.

A seleção significativa de enfermeiros deve estar relacionada ao fato do curso ser sediado em uma Escola de Enfermagem e receber a denominação de Enfermagem em Saúde Pública. Outro fator refere-se à necessidade de capacitar enfermeiros para atender ao processo de reordenamento do Sistema Nacional de Saúde desencadeado a partir da década de oitenta, com a ampliação da capacidade instalada da rede pública no nível de atendimento primário, dentro da proposta de descentralização dos serviços. E ainda, contarmos no Campus de Ribeirão Preto, com pós-graduação em Medicina só para médicos, em várias sub-áreas, inclusive em Medicina Social.

Também consideramos significativo os 17 outros profissionais de saúde $(25,40 \%)$ que foram selecionados. Se considerarmos os alunos do Campus da USP de Ribeirão Preto e de outros cursos de pós-graduação matriculados, neste período de sete anos, nas disciplinas obrigatórias do curso (Políticas de Saúde e Enfermagem e Sociedade), a participação de outros profissionais da saúde, que não enfermeiros, torna-se ainda mais significativa, pois estes têm uma demanda grande para estas disciplinas.

Tabela 1 - Caracterização dos alunos do programa de mestrado de enfermagem em saúde pública EERP/USP, segundo a categoria profissional, 1991-1997

\begin{tabular}{|c|c|c|c|c|c|c|c|c|c|}
\hline \multirow[b]{2}{*}{ Profissão } & \multirow[b]{2}{*}{1991} & \multirow[b]{2}{*}{1992} & \multirow[b]{2}{*}{1993} & \multirow[b]{2}{*}{1994} & \multirow[b]{2}{*}{1995} & \multirow[b]{2}{*}{1996} & \multirow[b]{2}{*}{1997} & \multicolumn{2}{|c|}{ TOTAL } \\
\hline & & & & & & & & $\mathrm{N}^{0}$ & $\%$ \\
\hline Enfermeiro & 05 & 09 & 04 & 07 & 06 & 11 & 08 & 50 & 74,60 \\
\hline Psicólogo & -- & 01 & -- & -- & -- & 01 & -- & 02 & 2,99 \\
\hline Odontólogo & -- & -- & 01 & 02 & -- & -- & -- & 03 & 4,47 \\
\hline AssistênciaSocial & -- & -- & 01 & -- & -- & -- & -- & 01 & 1,50 \\
\hline Fisioterapia & -- & -- & -- & -- & 01 & 01 & -- & 02 & 2,99 \\
\hline Nutricionista & -- & -- & -- & 01 & 02 & -- & 02 & 05 & 7,46 \\
\hline Farmac êutico & -- & -- & -- & -- & -- & 01 & -- & 01 & 1,50 \\
\hline Educador Físico & -- & -- & -- & -- & 01 & 01 & -- & 02 & 2,99 \\
\hline Fonoaudiólogo & -- & -- & -- & -- & -- & 01 & -- & 01 & 1,50 \\
\hline TOTAL & 05 & 10 & 06 & 10 & 10 & 16 & 10 & 67 & 100,00 \\
\hline
\end{tabular}

Fonte: Secretaria do Departamento de Enfermagem Materno-Infantil e Saúde Pública e Seção de Pós-Graduação da EERP-USP

Na Tabela 2 podem ser visualizados os itens (região geográfica, o vínculo empregatício e a atividade profissional) dos alunos. Assim verifica-se que a região sudeste predominou com 51 alunos $(76,0 \%)$ e os demais provém de vários outros estados, cobrindo todas as demais regiões do país.

Quanto ao vínculo empregatício constata-se que $71,60 \%$ dos alunos exercem suas atividades em instituições públicas. Este significativo percentual pode ser atribuído ao incentivo de algumas instituições públicas que estimulam a capacitação profissional para a progressão na carreira.

A atividade profissional de assistência é predominante com $46,27 \%$ de alunos, com uma diferença percentual de $4,47 \%$ de atividade de ensino $(41,80 \%)$.

Esta demanda de profissionais não docentes é uma realidade de todos os Cursos de Mestrado da pós-graduação em enfermagem da Escola de Enfermagem de Ribeirão Preto (MENDES et al., 1993), e também ocorre em cursos de outras instituições, bem como nos cursos das áreas de saúde pública e saúde coletiva.

Acredita-se que a predominância do número de mestrandos provenientes da área assistencial esteja relacionado ao reordenamento do setor saúde brasileiro, a partir dos anos 80 que vem conformando uma ampla rede básica de serviços de saúde, com a restruturação e investimento na vigilância epidemiológica e sanitária, na rede de informação, no planejamento e na gestão dos serviços, dentre outros, e com a preocupação da capacitação dos profissionais de saúde para atenderem a novas perspectivas de modelos de saúde mais voltados para as ações coletivas. 
Tabela 2 - Caracterização dos alunos do programa de mestrado de enfermagem em saúde pública EERP/USP, segundo a região geográfica, o vínculo e atividade profissional, 1991-1997

\begin{tabular}{|c|c|c|c|c|c|c|c|c|c|}
\hline \multirow[t]{2}{*}{ ANO } & \multirow[t]{2}{*}{1991} & \multirow[t]{2}{*}{1992} & \multirow[t]{2}{*}{1993} & \multirow[t]{2}{*}{1994} & \multirow[t]{2}{*}{1995} & \multirow[t]{2}{*}{1996} & \multirow[t]{2}{*}{1997} & \multicolumn{2}{|c|}{ TOTAL } \\
\hline & & & & & & & & $N^{0}$ & $\%$ \\
\hline \multicolumn{8}{|c|}{ REGIÃO } & \multirow[b]{2}{*}{01} & \multirow[b]{2}{*}{1,50} \\
\hline Norte & -- & -- & -- & -- & -- & 01 & -- & & \\
\hline Sul & -- & 01 & -- & -- & 02 & 02 & -- & 05 & 7,50 \\
\hline Sudeste & 04 & 07 & 04 & 10 & 07 & 11 & 08 & 51 & 76,00 \\
\hline Nordeste & 01 & 01 & -- & -- & -- & -- & -- & 02 & 3,00 \\
\hline Centro-Oeste & -- & 01 & 02 & -- & 01 & 02 & 02 & 08 & 12,00 \\
\hline Sub-total & 05 & 10 & 06 & 10 & 10 & 16 & 10 & 67 & 100,00 \\
\hline
\end{tabular}

VÍNCULO INSTITUCIONAL

\begin{tabular}{lccccccc|c|c}
\hline Instituição Pública & 04 & 06 & 06 & 06 & 08 & 11 & 07 & 48 & 71,60 \\
Instituição Privada & 01 & 03 & -- & 01 & 02 & 02 & 03 & 12 & 17,90 \\
Pública e Privada & -- & -- & -- & 02 & -- & -- & -- & 02 & 3,00 \\
Consultório Particular & -- & 01 & -- & -- & -- & 02 & -- & 03 & 4,50 \\
Sem Vínculo & -- & -- & -- & 01 & -- & 02 & -- & 03 & 4,50 \\
\hline Sub-total & 05 & 10 & 06 & 10 & 10 & \multirow{2}{*}{16} & \multirow{2}{*}{10} & 67 & 100,00 \\
\hline
\end{tabular}

ATIVIDADE PROFISSIONAL

\begin{tabular}{llllllll|l|l}
\hline Ensino & 02 & 06 & 03 & 02 & 05 & 06 & 04 & 28 & 41,80 \\
Assistência & 03 & 02 & 03 & 05 & 05 & 07 & 06 & 31 & 46,27 \\
Ensino/Assistência & -- & 01 & -- & 02 & -- & -- & -- & 03 & 4,47 \\
Outros & -- & 01 & -- & 01 & -- & 03 & -- & 05 & 7,46 \\
\hline Sub-total & 05 & \multirow{2}{*}{10} & 06 & 10 & 10 & \multirow{2}{*}{16} & \multirow{2}{*}{10} & 67 & 100,00 \\
\hline
\end{tabular}

Fonte: Secretaria do Departamento de Enfermagem Materno-Infantil e Saúde Pública e Secretaria da Pós-Graduação da EERPUSP

\subsection{Caracterização e análise das dissertações quanto aos objetos de estudo e suas relações com os problemas oriundos das práticas em saúde}

Os recortes dos objetos e as metodologias das investigações comportam formas distintas de análise, desde diagnósticos de situações que enfatizam aspectos quanti-qualitativos, bem como avaliação de programas com propostas de intervenções. Relacionam também os objetos com o contexto político-social, os modelos assistenciais de saúde, ao movimento de reformulação das práticas de saúde e as representações sociais do processo saúde-doença, de tal forma que o recorte dos problemas possa dar conta dos aspectos operativos das práticas de saúde e os seus reflexos no cotidiano dos serviços.

Quanto às áreas temáticas das dissertações, tabela 3, destaca-se primeiramente que a maioria dos objetos de estudo, em número de 12, está inserida em organização da assistência em saúde. Referem-se a aspectos específicos dos programas de imunização como levantamento de faltosos, avaliação de cobertura vacinal e do programa de imunização no processo de municipalização da saúde, e também temáticas que adotam estudos epidemiológicos tais como doenças crônico-degenerativas e hipertensão arterial; trabalhos que procuram analisar a organização do trabalho e da assistência à saúde, perpassando os distintos saberes e práticas que a constituem, envolvendo estudos de avaliação da assistência prestada a determinada clientela (mulher, mulher-gestante e crianças com deficiência física e mental). Outra temática estudada na área de políticas de saúde, sobre o processo de municipalização/ descentralização e do movimento social organizado em regiões brasileiras diferenciadas (nordeste, sudeste e sul), retratando a preocupação de estudar realidades concretas, suscitando novos realinhamentos nos recortes dos objetos de investigação que permitam propor estratégias de intervenção na prática cotidiana dos serviços de saúde.

O processo saúde-doença é um outro eixo 
temático presente em 06 dissertações analisadas, que busca a relação entre a enfermagem e o campo de conhecimento específico da saúde pública tais como tuberculose, AIDS, hanseníase e as abordam em questões concernentes à organização do trabalho, além de tentar criar novas formas de interpretar as determinações do processo saúde-doença com enfoque de estilo de vida e de fatores de riscos que o caracterizam.

A área temática de promoção à saúde, com 05 dissertações, aborda análises teórico-conceituais sobre os paradigmas na saúde, a qualidade de vida e as representações sociais dos trabalhadores de saúde e dos usuários dos serviços, com recortes bem delimitados e voltados para problemas específicos, a exemplo de gravidez na adolescência e a representação social de mulheres de uma região periférica de um determinado município, acerca de suas demandas e experiências no campo da saúde.
A nutrição/alimentação, com 06 dissertações constitui-se em um tema importante que procura analisar os programas assistenciais de aleitamento materno e de suplementação alimentar às clientelas atendidas nas unidades básicas de saúde e na rede escolar de municípios brasileiros.

Ainda há uma dissertação que enfatiza a prática profissional da enfermeira na perspectiva das relações de gênero e do poder constituído dentro do espaço institucional hospitalar. Como também um estudo experimental que avalia aspectos imunológicos da vacina anti-rábica.

A análise dos objetos de pesquisa permite concluir que há uma preocupação dos autores em retratar as realidades vivenciadas por eles e traçar estratégias de intervenção no espaço micro, onde se efetivam as práticas, nas áreas de conhecimento da saúde da mulher, da criança e do adolescente e da saúde pública propriamente dita.

Tabela 3 - Distribuição das áreas temáticas e das áreas de conhecimento das dissertações do programa de mestrado em enfermagem de saúde pública da EERP-USP, 1993-1997

\begin{tabular}{|c|c|c|c|c|c|c|c|}
\hline $\begin{array}{l}\text { AREAS } \\
\text { TEMAMTIC AS }\end{array}$ & $\begin{array}{l}\text { ORGANIZACÃO } \\
\text { DA ASSISTÊHCIA } \\
\text { ÂSAÚDE }\end{array}$ & $\begin{array}{l}\text { PROCESSC } \\
\text { SAUUDE } \\
\text { DOENÇA }\end{array}$ & $\begin{array}{l}\text { PROMOÇ̃̃O } \\
\text { A SAÚDE }\end{array}$ & $\begin{array}{l}\text { NUTRIÇÄOI } \\
\text { ALIMENTAÇO }\end{array}$ & $\begin{array}{l}\text { PRÁTICA } \\
\text { PROFISSIONAL }\end{array}$ & IMUNOLOGIA & TOTAL \\
\hline \multicolumn{8}{|l|}{$\begin{array}{l}\text { AREAS DE } \\
\text { CONHECIMENTO }\end{array}$} \\
\hline $\begin{array}{l}\text { Enf. em Saúde da } \\
\text { Mulher }\end{array}$ & 02 & 01 & 02 & 02 & 01 & -- & 08 \\
\hline $\begin{array}{l}\text { Enf. em Saúde da } \\
\text { Criança e } \\
\text { Adolescente }\end{array}$ & 03 & 01 & 02 & 04 & -- & -- & 10 \\
\hline Enf. de Saúde Pública & 07 & 04 & 01 & -- & -- & 01 & 13 \\
\hline TOTAL & 12 & 06 & 05 & 06 & 01 & 01 & 31 \\
\hline
\end{tabular}

Fonte: Dissertações de Mestrado defendidas no período de 1993 a 1997 do Programa de Mestrado de Enfermagem em Saúde Pública da EERP-USP

\section{4 - CONCLUSÕES}

As dissertações ao delimitarem problemas concretos do cotidiano tecem propostas de intervenção referentes a organização dos serviços e ao modelo assistencial, na área de saúde da mulher, da criança e do adolescente, bem como se voltam para questões específicas que envolvem os programas e problemas de saúde pública como vacinação, tuberculose, hanseníase, doenças crônicodegenerativas, dentre outros.

Ao repensar e criticar os saberes e as práticas de saúde, na sua dinâmica social e histórica, o Programa de Mestrado estimula a produção de conhecimentos voltados para temáticas emergentes, como a crise de paradigmas na saúde, as relações de gênero e saúde e os movimentos sociais. A doença é estudada na confluência do biológico e social e a prática em saúde além de técnica é tomada como prática social.
A produção científica dos programas de pós-graduação da área de saúde coletiva no Brasil, a partir da década de 70, mesmo sendo heterogênea, guarda uma base comum que é trazer o social para compreender o processo saúde-doença, as práticas e os saberes (NUNES, 1996).

$\mathrm{O}$ fato da pesquisa em enfermagem ser de natureza aplicada facilita que os recortes dos objetos de investigação de sua produção científica sejam aproximados dos problemas das práticas de saúde.

A análise dos objetos de pesquisa do Mestrado de Enfermagem em Saúde Pública evidencia uma articulação entre a universidade e o movimento da reforma sanitária e, no caso, do Departamento MISP que tem uma significativa postura política na adoção de estratégias de extensão de serviços à comunidade, como fonte de intercâmbio entre teoria e prática, universidade e trabalho.

Esta articulação universidade \& serviços que é retratada no produto do Programa de Pós-Graduação, tem 
sido um fator estimulador para a continuidade da formação dos profissionais oriundos de universidades e de serviços, como pode ser verificado que cerca da metade dos alunos

é proveniente dos serviços de saúde e metade da docência.

\section{KNOWLEDGE PRODUCED IN THE MASTER PROGRAM ON PUBLIC HEALTH NURSING OFFERED BY THE UNIVERSITY OF SÃO PAULO AT RIBEIRÃO PRETO COLLEGE OF NURSING AND ITS RELATIONSHIP WITH HEALTH PRACTICES}

The purpose of this study was to analyze the research objects of the theses produced in the Master Program on Public Health offered by the Department of Maternal-Infant Nursing and Public Health - University of São Paulo at Ribeirão Preto College of Nursing and their relationship with the problems originated from health care practice. These practices were classified within six major areas: Health Care Organization, Health Promotion, Health-Disease Process, Nutrition-Feeding, Professional Practice and Immunology. The contribution of works focussing on the crisis of health paradigms, gender relations and Social Movements in Health were emphasized. Authors concluded that the themes are closely related to the students' professional context, enabling them to present suggestions regarding interventions in health practices.

KEY WORDS: public health nursing, education, nursing, graduate nursing education, public health practice

\section{EL CONOCIMIENTO PRODUCIDO EN EL PROGRAMA DE MAESTRÍA DE ENFERMERÍA EN SALUD PÚBLICA DE LA ESCUELA DE ENFERMERÍA DE RIBEIRÃO PRETO-USP Y SU RELACIÓN CON LAS PRÁCTICAS DE SALUD}

Se analizan los objetos de investigación de tesis de maestría en Enfermería en Salud Pública del MISP de EERP, y sus relaciones con problemas originados de las prácticas de salud. Estas fueron clasificadas en seis áreas temáticas: Organización Asistencial en Salud, Promoción de Salud, Proceso Salud Enfermedad, Nutrición-Alimentación, Práctica Profesional e Inmunología. Se destaca la contribución de trabajos con respecto a la crisis de los paradigmas en salud, relaciones de género y los movimientos sociales en salud. Se concluyó, que las temáticas reflejan realidades profesionales de los alumnos con propuestas de intervención en el micro-espacio de las prácticas de salud.

TÉRMINOS CLAVES: enfermería en salud pública, educación de postgrado en enfermería, práctica de salud pública

\section{REFERÊNCIAS BIBLIOGRÁFICAS}

01. ALMEIDA, M.C.P.de et al. A pesquisa no ensino de pós-graduação em enfermagem stricto sensu. In: SEMINÁRIO NACIONAL DE PESQUISA EM ENFERMAGEM, 8., Ribeirão Preto, 1995. Anais. Ribeirão Preto: Associação Brasileira de Enfermagem, 1995. p. 16-32.

02. ESCOLA DE ENFERMAGEM DE RIBEIRÃO PRETO. Departamento de Enfermagem Materno Infantil e Saúde Pública. Proposta de criação do Programa de Mestrado de Enfermagem de Saúde Pública. Ribeirão Preto: Escola de Enfermagem de Ribeirão Preto, 1990. / mimeografado/

03. JEFFRIES, N. Converging educational perspectives. New York: National League for Nursing, 1996. 303p.
04. MENDES, I.A.C.; GIR, E.; TREVIZAN, M.A. Escola de Enfermagem de Ribeirão Preto da Universidade de São Paulo: Centro Colaborador da Organização Mundial de Saúde. Rev.Latinoam.Enfermagem, v. 1, p. 52-68, dez. 1993. n.especial.

05. MORYIA, T.M. et al. Pós Graduação stricto sensu em enfermagem: um estudo do seu desenvolvimento no Brasil. Ribeirão Preto: Fundação Instituto de Enfermagem de Ribeirão Preto, 1998. 189p.

06. NUNES, E.D.. Saúde coletiva: revisitando a sua história e os cursos de pós-graduação. Ciência \& Saúde Coletiva, v. 1, n. 1, p. 55-69, 1996.

07. WRIGHT, M.da G.; ALARCÓN, N.G. Organización Panamericana de la Salud: estudio de los programas de especialización y maestria en enfermería en América Latina. In: JEFFRIES, Nancy. Converging educational perspectives. New York: National League for Nursing, 1996. 